\title{
A NOTE ON A THEOREM OF PERRON
}

\author{
K. C. PRASAD AND M. LARI
}

\begin{abstract}
Given an infinite simple continued fraction $\left[a_{0}, a_{1}, \ldots, a_{n}, \ldots\right]$, let $M_{n}$ denote $\left[0, a_{n}, a_{n-1}, \ldots, a_{1}\right]+\left[a_{n+1}, a_{n+2}, \ldots\right]$. A well-known result due to Perron [1, III, 212] states: If $a_{n+2}=m$, then there is a $k$ in $\{n, n+1, n+2\}$ for which $M_{k}>\sqrt{m^{2}+4}$. In this note we give a new proof for this result and add that there is a $j$ in $\{n, n+1, n+2\}$ for which $M_{j}<\sqrt{m^{2}+4}$.
\end{abstract}

1. Introduction. Let $x$ be an irrational number whose simple continued fraction expansion is $\left[a_{0}, a_{1}, a_{2}, \ldots\right]$. It is well known [1, Chapter III, 1(6)] that $\mid x-$ $p_{n} / q_{n} \mid=1 / M_{n} q_{n}^{2}$, where $p_{n} / q_{n}=\left[a_{0}, a_{1}, \ldots, a_{n}\right]$ and $M_{n}=\left[0, a_{n}, a_{n-1}, \ldots, a_{1}\right]+$ $\left[a_{n+1}, a_{n+2}, \ldots\right]$. The following result, essentially due to Perron [1, III, 2.12], has received much attention:

$$
\begin{aligned}
& \text { If } a_{n+2}=m \text {, then } M_{k}>\sqrt{m^{2}+4} \text { for at least one value } \\
& \text { of } k \text { in }\{n, n+1, n+2\} \text {. }
\end{aligned}
$$

This note displays a simple strategy which leads to this result and also unfolds another interesting fact. We prove the following

THEOREM. If $a_{n+2}=m$, then there is a $k$ in $\{n, n+1, n+2\}$ for which $M_{k}>$ $\sqrt{m^{2}+4}$, and there is a $j$ in $\{n, n+1, n+2\}$ for which $M_{j}<\sqrt{m^{2}+4}$.

2. Proof of the Theorem. Set $a_{n+2}=m, u=\sqrt{m^{2}+4}, s=\left[0, a_{n+3}, a_{n+4}\right.$, $\ldots]$, and $t=\left[a_{n+1}, a_{n}, \ldots, a_{1}\right]$. Now $M_{n}=t+1 /(m+s), M_{n+1}=1 / t+m+s$ and $M_{n+2}=t /(m t+1)+1 / s$. Again put $h_{1}(t)=1 /(u-t)-m, h_{2}(t)=u-m-1 / t$ and $h_{3}(t)=(m t+1) /((u m-1) t+u)$. Also

$$
h_{2}(t)-h_{1}(t)=-\frac{u}{t(u-t)}\left(t-\frac{u+m}{2}\right)\left(t-\frac{u-m}{2}\right)
$$

and

$$
h_{3}(t)-h_{2}(t)=-\frac{u\left(m u-m^{2}-1\right)}{t(m u t-t+u)}\left(t-\frac{u+m}{2}\right)\left(t+\frac{3 m+u}{2\left(2 m^{2}-1\right)}\right) .
$$

Note that $(u-m) / 2<1 \leq t$ and $u \neq t$ because $t$ is rational and $u$ is not. The following cases appear:

Case 1. $u>t$ and $t<(u+m) / 2$. In this case

$$
\begin{aligned}
& \operatorname{Sign}\left\{h_{2}(t)-h_{1}(t)\right\}=(-) \cdot(-) \cdot(+)=(+), \\
& \operatorname{Sign}\left\{h_{3}(t)-h_{2}(t)\right\}=(-) \cdot(-) \cdot(+)=(+) .
\end{aligned}
$$

So $h_{1}(t)<h_{2}(t)<h_{3}(t)$. Thus if $s \leq h_{1}(t)$, then we have $s<h_{2}(t)$ and $s<h_{3}(t)$, which are equivalent to $M_{n+1}<u$ and $M_{n+2}>u$, respectively. Or if $h_{1}(t)<s \leq$

Received by the editors March 5, 1985 and, in revised form, May 31, 1985.

1980 Mathematics Subject Classification. Primary 10Fxx; Secondary 10F05. 
$h_{2}(t)$, then we have $h_{1}(t)<s$ and $s<h_{3}(t)$, which are equivalent to $M_{n}<u$ and $M_{n+2}>u$, respectively. Or if $h_{2}(t)<s$, then we have $h_{1}(t)<s$ and $h_{2}(t)<s$, which are equivalent to $M_{n}<u$ and $M_{n+1}>u$, respectively.

Case 2. $u>t$ and $(u+m) / 2<t$. In this case we get $h_{3}(t)<h_{2}(t)<h_{1}(t)$ and argue as above.

Case 3. $u<t$. In this case $h_{1}(t)<0<s$ or $M_{n}>u$. Also $(u+m) / 2<u<t$. Therefore $\operatorname{Sign}\left\{h_{3}(t)-h_{2}(t)\right\}=(-)$. So if $s \leq h_{3}(t)$, we get $s<h_{2}(t)$ or $M_{n+1}<u$. Otherwise we have $h_{3}(t)<s$ or $M_{n+2}<u$.

This completes the proof of the theorem.

We are thankful to the referee for his helpful comments.

\section{REFERENCES}

1. J. F. Koksma, Diophantische Approximationen, Chelsea, New York, 1936.

2. E. M. Wright, Approximation of irrationals by rationals, Math. Gaz. 48 (1964), 288-289.

3. F. Bagemihl and J. R. McLaughlin, Generalisations of some classical theorems concerning triples of consecutive convergents to simple continued fractions, J. Reine Angew. Math. 221 (1966), 146-149.

DePaRtMent of Mathematics, RANCHi University, RANCHI, INDia

A.E.T.-I.M.D., Dry LAND Agriculture Research Centre, Birsa AgRiculture UNIVERSITY, KANKE, RANCHI 834006, INDIA 